\title{
Proton-induced pre-equilibrium composite-particle emission
}

\author{
Anthony Cowley ${ }^{1, *}$ \\ ${ }^{1}$ Department of Physics, Stellenbosch University, Private Bag X1, Matieland 7602, South Africa
}

\begin{abstract}
The reaction mechanism of proton-induced composite particle pre-equilibrium emission as an intrinsic part a statistical multistep process was confirmed long ago. Nevertheless, until fairly recently it was still not clear how pickup and knockout processes compete in the multistep sequence to produce a composite ejectiles. An overview of results and conclusions based on recent $(p, \alpha)$ studies at incident energies from 65 to $200 \mathrm{MeV}$ are presented and evaluated. It is shown how analyzing power is a valuable observable for unravelling the details of the reaction mechanism. Future avenues of investigation are discussed.
\end{abstract}

\section{Introduction}

Insight into the reaction mechanism of proton-induced pre-equilibrium $\alpha$-particle emission has progressed considerably during the last 7 years as a result of investigations conducted by an international collaboration from Stellenbosch, Sofia and Dubna [1-6]. The purpose of this review is to highlight how these new perspectives which emerged from our studies contrast with long-held misconceptions of how the reaction mechanism behaves, and why the earlier incorrect viewpoints took a foothold in the past.

The exciton model [7] of Griffin is an early classical theoretical description of how an energetic nucleon projectile interacts with nuclei in general to cause stepwise excitations of the intermediate nuclear system. In this model, at each stage of the successive steps of the interaction, the relevant system is characterized by an increasingly complex structure, and emission of nucleons and composite ejectiles is energetically possible. One of the early experiments to demonstrate the veracity of the theoretical ideas was the work of Bertrand and Peelle [8]. This work was soon followed by numerous further experimental studies and simultaneous development of quantum mechanical formulations of a statistical multistep theory. Examples of roughly equivalent quantum mechanical theories are those of Feshbach, Kerman and Koonin [9], Tamura, Udagawa and Lenske [10], and Nishioka, Weidenmüller and Yoshida [11]. Koning and Akkermans [12] compare these theoretical implementations of basically the same mechanism with each other and they also describe the relationships between the different formulations. The earliest studies successfully described $\left(p, p^{\prime}\right)$ pre-equilibrium reactions. At an early stage it was also recognized that emission of composite ejectiles in pre-equilibrium reactions should follow the same basic multistep mechanism,

\footnotetext{
*e-mail: aac@sun.ac.za
}

with differences due to details of the interaction of composite particles with nuclear matter.

\section{Multistep statistical model}

Of the available quantum mechanical formulations, the version of Feshbach, Kerman and Koonin (FKK) [9] has found most application, and consequently this is also used in our work. The double-differential cross section is expressed [13] in terms of solid angle $d \Omega$ and emission energy $d E$ acceptance as

$$
\frac{d^{2} \sigma}{d \Omega d E}=\left(\frac{d^{2} \sigma}{d \Omega d E}\right)^{1-s t e p}+\left(\frac{d^{2} \sigma}{d \Omega d E}\right)^{2-s t e p}+\cdots
$$

where the first step cross section is taken as a direct reaction calculated in terms of the DWBA. This term is given by

$$
\left(\frac{d^{2} \sigma}{d \Omega d E}\right)^{1-\text { step }}=\sum_{N, L, J} \frac{(2 J+1)}{\Delta E} \frac{d \sigma^{D W}}{d \Omega}(\theta, N, L, J, E),
$$

at scattering angle $\theta$, where the summation runs over the target states with single-particle energies within a small interval $(E-\Delta E / 2, E+\Delta E / 2)$ around the excitation energy $E$. If the DWBA calculation is treated as a knockout, quantum numbers $N, L$ and $J$ refer to the $\alpha$-cluster bound in the target, otherwise to those of the three-nucleon system which is picked up. The differential cross sections $\frac{d \sigma^{D W}}{d \Omega}$ to particular $(N, L, J)$ states are calculated using the code DWUCK4 [14].

The multistep cross sections, which are appropriate for the second and higher steps of the $(p, \alpha)$ reaction, are ex- 


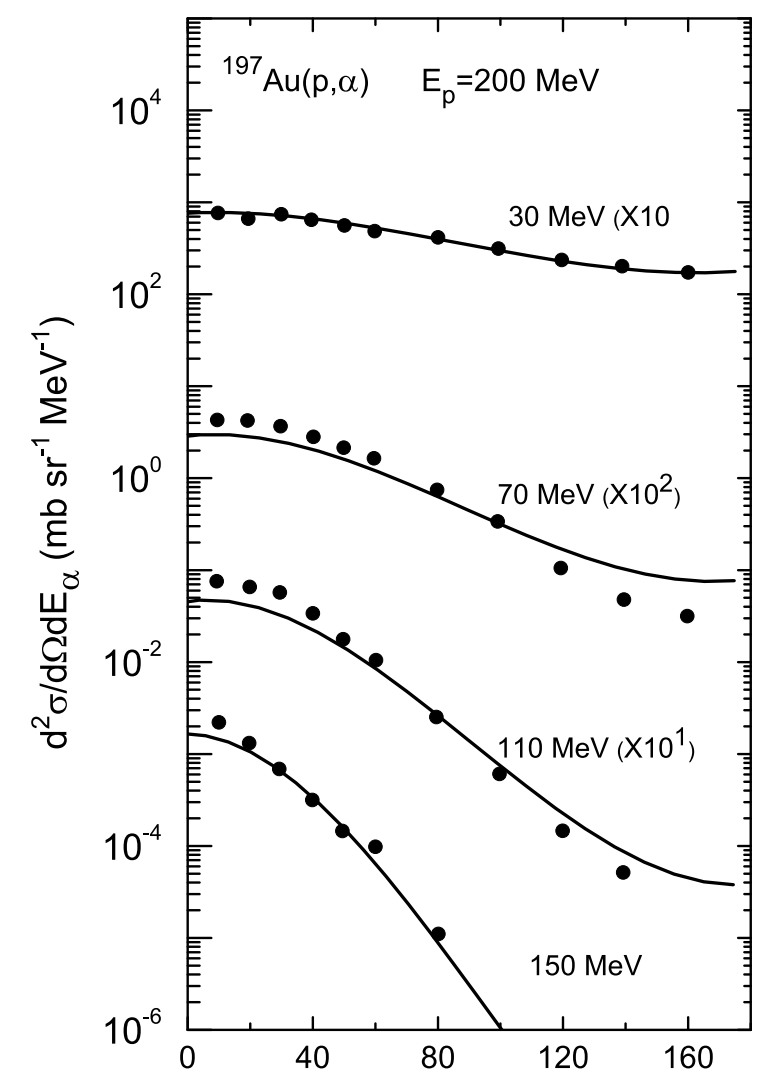

Figure 1. Cross section angular distributions for the reaction ${ }^{197} \mathrm{Au}(p, \alpha)$ at an incident energy of $200 \mathrm{MeV}$. Ejectile energies are shown with each distribution and cross sections are multiplied by the indicated factors for clarity of display. The curves are predictions of the systematics expressed in Eq. 4. Results are adapted from Cowley et al. [15].

pressed as

$$
\begin{aligned}
& \left(\frac{d^{2} \sigma}{d \Omega d E}\right)^{\text {multistep }}= \\
& \sum_{n=2}^{n_{\max }} \sum_{m=n-1}^{n+1} \int \frac{d \vec{k}_{1}}{(2 \pi)^{3}} \int \frac{d \vec{k}_{2}}{(2 \pi)^{3}} \ldots \int \frac{d \vec{k}_{n}}{(2 \pi)^{3}} \\
& \times\left(\frac{d^{2} \sigma\left(\vec{k}_{f}, \vec{k}_{n}\right)}{d \Omega_{f} d E_{f}}\right) \times\left(\frac{d^{2} \sigma\left(\vec{k}_{n}, \vec{k}_{n-1}\right)}{d \Omega_{n} d E_{n}}\right) \times \ldots \\
& \times\left(\frac{d^{2} \sigma\left(\vec{k}_{2}, \vec{k}_{1}\right)}{d \Omega_{2} d E_{2}}\right) \times\left(\frac{d^{2} \sigma\left(\vec{k}_{1}, \vec{k}_{i}\right)}{d \Omega_{1} d E_{1}}\right)_{p, p^{\prime}}^{1-s t e p}
\end{aligned}
$$

where $\vec{k}_{i}, \vec{k}_{n}$ and $\vec{k}_{f}$ are the momenta of the initial, $n^{\text {th }}$ and final steps. The number of reaction steps is indicated with the symbol $n$, the maximum number of reaction steps is $n_{\text {max }}$ and $m$ is the exit mode. Therefore the cross section associated with $m$ is given leading to the emission of an $\alpha$-particle, and all steps prior to the final step are nucleonnucleon collisions.

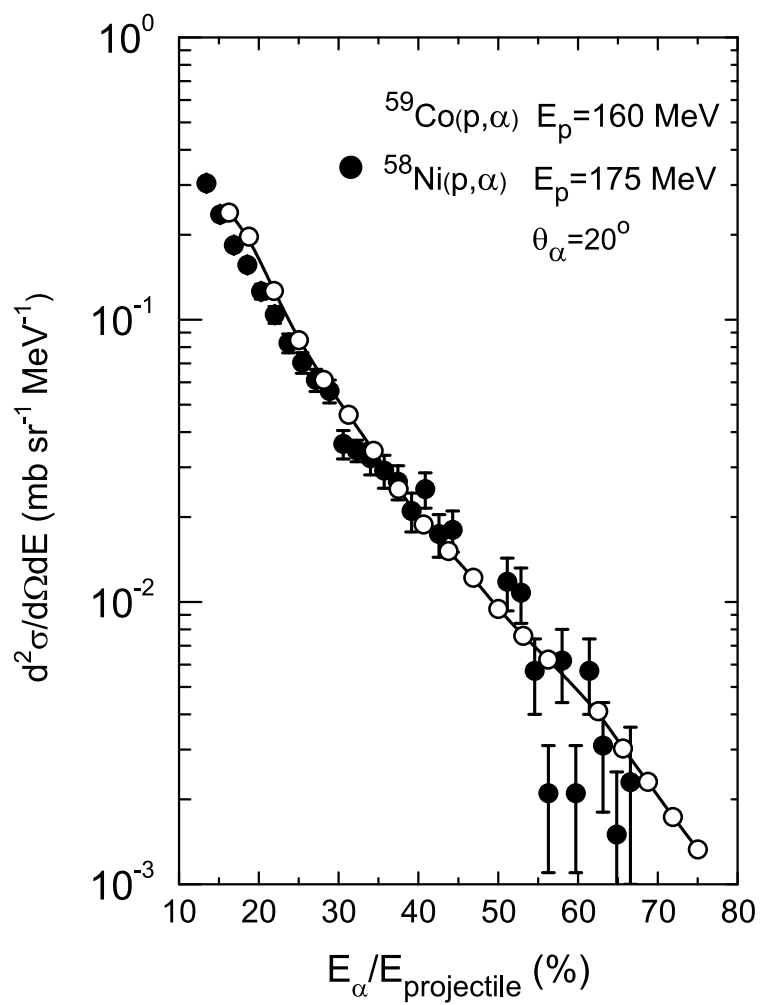

Figure 2. Comparison between cross section distribution for the reaction ${ }^{59} \mathrm{Co}(p, \alpha)[16]$ and ${ }^{58} \mathrm{Ni}(p, \alpha)$ [17] at incident energies of 160 and $175 \mathrm{MeV}$, respectively. Results are displayed as a function of the ratio of emitted $\alpha$-particle energy to the incident energy of the projectile. Figure from Cowley [18].

\section{Target-mass and incident-energy trend of pre-equilibrium cross sections}

Because of the convoluted reaction mechanism theoretically expected for a pre-equilibrium process, strong target mass dependence or incident energy variation of the cross section distributions would not be expected. This expectation seems to be supported by several experimental studies of $\left(p, p^{\prime}\right)$ pre-equilibrium reactions. (See for example [19]). Further support for insensitivity to target mass or incident energy is provided by the phenomenological expression developed by Kalbach [20]. This formulation successfully describes the angular variation of the preequilibrium cross section $\frac{d^{2} \sigma}{d \Omega d E_{b}}$ with emission energy $E_{b}$ as

$$
\frac{d^{2} \sigma}{d \Omega d E_{b}}=\sigma_{D} \frac{\eta}{\sinh \eta} \exp (\eta \cos \theta)
$$

where $4 \pi \sigma_{D}$ is the total angle-integrated cross section for a specific type of pre-equilibrium reaction, $\theta$ is the scattering angle and $\eta$ is a slope parameter which is a simple power function of $E_{b}$.

This distribution implies that, at high emission energy, the cross section drops steeply with $\theta$, as would be expected for the highly-direct nature of the initial stage of the reaction chain. As the emission energy drops, the rate 


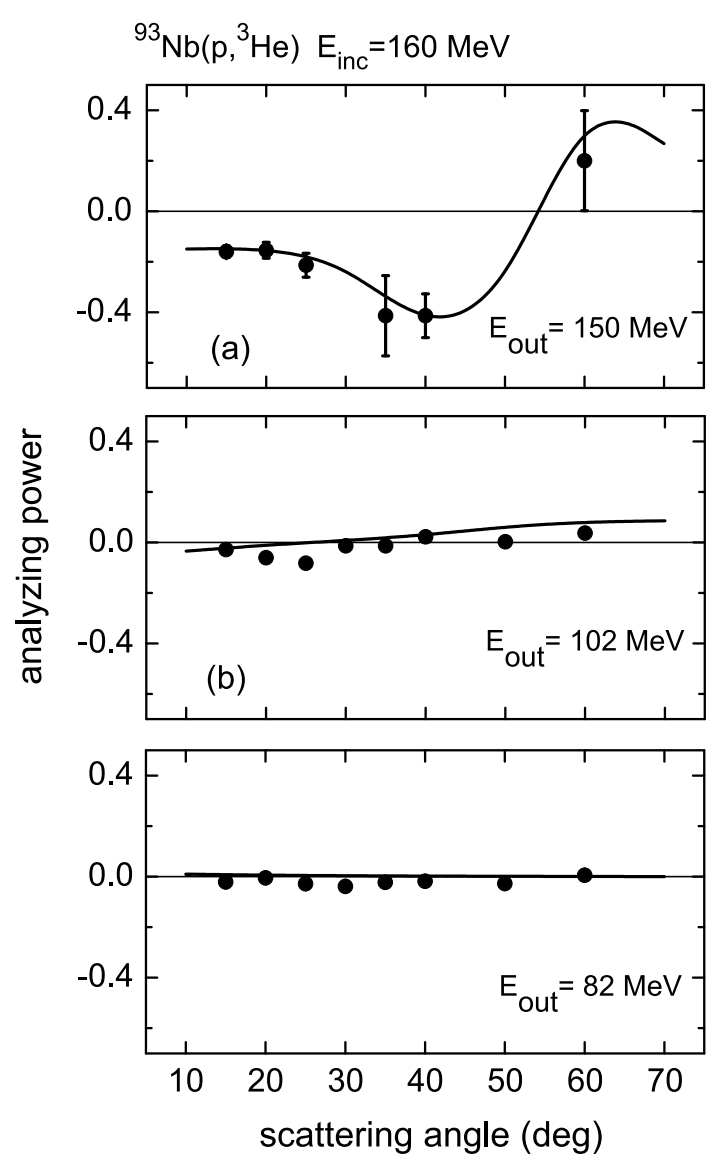

Figure 3. Analyzing power angular distributions for the reaction ${ }^{93} \mathrm{Nb}\left(p,{ }^{3} \mathrm{He}\right)$ at an incident energy of $160 \mathrm{MeV}$ and emission energies as indicated. The curves are predictions of the FKK theory. Figure adapted from Dimitrova et al. [3].

of decrease with $\theta$ of the cross section becomes less prominent. This reflects the growth of number of stages of the reaction process, which eventually would reach an isotropic (or symmetric around $90^{\circ}$ ) angular distribution as equilibration is reached. A typical example of how accurately the Kalbach [20] formula reproduces experimental angular distributions is shown in Fig. 1.

The simple form of the parametrisation of the cross section angular distribution hints at an equally simple fundamental relationship of the relevant reaction mechanisms which are shared between any species of light projectile or ejectile. Also, the phenomenological properties of the crucial parameter $\eta$ confirm the idea that the cross section distributions would not be very sensitive to, for example, detailed structure of the target mass. The relative insensitivity to projectile energy and target is, for example, illustrated in Fig. 2 where a simple energy rescaling of the two sets of experimental distributions accounts for the difference between the data.

\section{Analyzing power angular distributions}

The analyzing power is expressed as

$$
A_{y}=\frac{\sigma_{L}-\sigma_{R}}{\sigma_{L}+\sigma_{R}}
$$

where $\sigma_{L}$ and $\sigma_{R}$ are cross section yields defined in directions to the left and to the right in a coordinate system which has the Z-axis in the direction of the projectile momentum, and with the projectile polarized perpendicular to the reaction plane defined by $\mathrm{X}-\mathrm{Z}$.

The multistep expression for the analyzing power develops as

$$
A_{\text {multistep }}=\frac{A_{1}\left(\frac{d^{2} \sigma}{d \Omega d E}\right)^{1-\text { step }}+A_{2}\left(\frac{d^{2} \sigma}{d \Omega d E}\right)^{2-\text { step }}+\cdots}{\left(\frac{d^{2} \sigma}{d \Omega d E}\right)^{1-s t e p}+\left(\frac{d^{2} \sigma}{d \Omega d E}\right)^{2-s t e p}+\cdots},
$$

with $A_{i},\{i=1,2, \ldots\}$ referring to analyzing powers for the successive multisteps. When different reaction mechanisms terminating each sequence of steps are involved, these are treated in an analogous way through the structure of this formulation.

Because the analyzing power is a ratio of cross sections, it is independent of the absolute cross section of the reaction. This is especially useful in the case of $(p, \alpha)$ cluster reactions, for which DWBA calculations are prone to a so-called model dependence because of the large momentum difference between the incident and exit channel for reasonably low Q-values. Momentum conservation restricts knocked out clusters to large momentum values, for which wave functions of exceptional accuracy are required for an accurate theoretical estimate of the absolute cross section.

As would be expected, it is found that the analyzing power angular distribution is much more sensitive to the multistep character of a pre-equilibrium reaction than a cross section angular distribution. Whereas the slopes of the cross section angular distributions change only in a subtle way with higher steps in the reaction stage [21], analyzing power distributions change shape rapidly, as is shown in Fig. 3. At the highest emission energy, where the first stage is dominant in Fig. 3, the analyzing power distribution shows large excursions from zero. This analyzing power value progressively settles at zero as higher stages of the reaction mechanism enter. Already when the second stage is most prominent at the lowest emission energy shown, the analyzing power remains essentially at zero over the whole angular range.

Another advantage of the analyzing power observable is that it offers a simple identification of the reaction mechanism in composite particle emission. For example, the analyzing power of $\left(p,{ }^{3} \mathrm{He}\right)$ reactions, without exception between incident energies of 100 and $160 \mathrm{MeV}$ $[1,16,22,23]$, is found to be consistent with two-nucleon pickup as the terminating process at each individual stage of the reaction. On the other hand, $(p, \alpha)$ reactions appear to experience competition between pickup and knockout, with the magnitude of the two processes varying depending on details of the reaction process [2-4].

Figure 4 shows examples of analyzing power angular distributions for $(p, \alpha)$ reactions on ${ }^{58} \mathrm{Ni}$ [24] and ${ }^{93} \mathrm{Nb}$ [4] at an incident energy of roughly $70 \mathrm{MeV}$ and an emission energy which is exactly $12 \mathrm{MeV}$ lower that that of the projectile. In both cases the theoretical prediction identifies the reaction mechanism as driven purely by a terminating 


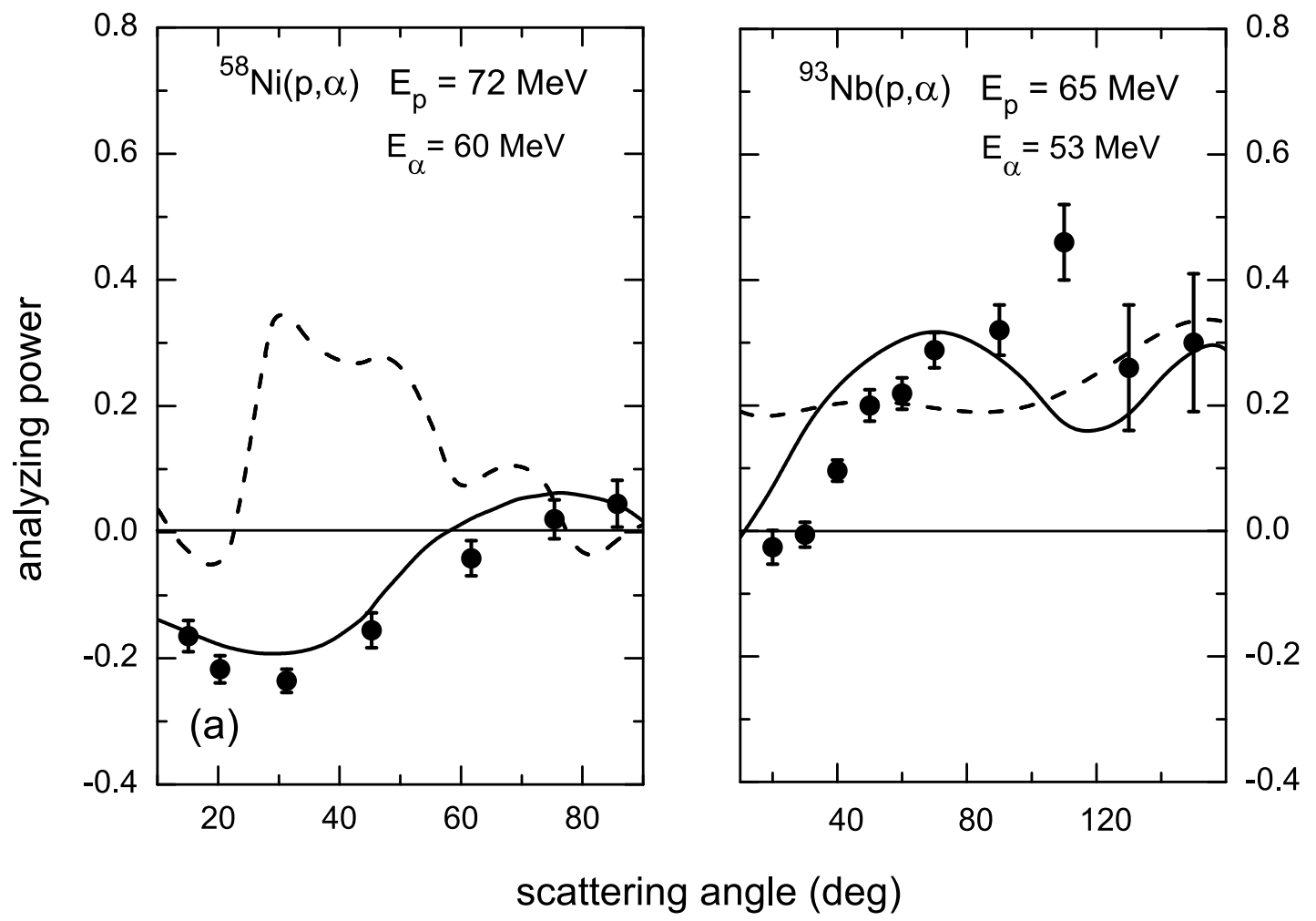

Figure 4. Analyzing power angular distributions for $(p, \alpha)$ reactions on (a) ${ }^{58} \mathrm{Ni}$ and (b) ${ }^{93} \mathrm{Nb}$ at roughly similar incident energies, as indicated. In both cases the emission energy is $12 \mathrm{MeV}$ lower than that of the projectile. A solid (dashed) curve indicates a knockout (pickup) mechanism as the ultimate step which leads to emission of an $\alpha$-particle. Results in (a) are from Bonetti et al. [24] and in (b) from Cowley et al. [4]. Experimental data in (a) are from Lewandowski et al. [25] and in (b) are from Sakai et al. [26].

knockout process. It is significant that for neither target does an alternative pickup mechanism contribute at all. In the case of ${ }^{58} \mathrm{Ni}$ the analyzing power for pickup is even of the wrong sign. For ${ }^{93} \mathrm{Nb}$ the trend of the analyzing power is also drastically wrong for a pickup mechanism.

It should be noted that, apart from the mass difference, ${ }^{58} \mathrm{Ni}$ and ${ }^{93} \mathrm{Nb}$ are very different nuclear species which differ in ground state spin and general structure. Presumably properties such as cluster preformation would also differ appreciably. Consequently the similarity of the pre-equilibrium reaction mechanism is not expected.

As was already mentioned, the $\left(p,{ }^{3} \mathrm{He}\right)$ preequilibrium reaction was found previously to proceed predominantly through a terminating pickup process over a large range of incident energies. Although it is reasonable to accept that a knockout process would be severly suppressed for $\left(p,{ }^{3} \mathrm{He}\right)$ reaction because of low ${ }^{3} \mathrm{He}$ clustering probability, the reason for the total absence of pickup for the $(p, \alpha)$ found around an incident energy of around $70 \mathrm{MeV}$ is not obvious. On the other hand, it was not a surprise to discover a dominance of pickup for ${ }^{93} \mathrm{Nb}(p, \alpha)$ at an incident energy of $100 \mathrm{MeV}$ [3, 4]. Nevertheless, that the dominant reaction mechanism could change so dramatically on increasing by such a small amount in incident energy as from 65 to 100 $\mathrm{MeV}$, is interesting. However, Cowley et al. [4] show that such behaviour is quite consistent with predicted trends of knockout and pickup in a distorted-wave Born approximation (DWBA). Of course, the DWBA is the main ingredient of the statistical multistep formulation.

The variation of the cross section of a pickup process with incident energy is determined [4] by the transferred angular momentum which maximizes the reaction yield at an energy value which corresponds to momentum matching between the incident and outgoing energy. Thus the cross section from the pickup process tends to increase up to some incident energy, and drops thereafter. Knockout, on the other hand, with only the struck cluster mostly emerging from the system, generally has no associated angular momentum transfer, and the knocked out cluster needs to provide momentum for it to be conserved overall [4]. This means that the yield based on a knockout process drops with increasing incident energy.

Clearly the relative cross section yield from knockout and pickup at any incident energy should depend on the details of the target system to a much greater extent than was appreciated up to now. Therefore it is not surprising to sometimes encounter evidence of a competition of knockout and pickup in $(p, \alpha)$ pre-equilibrium reactions. An example of such a case is shown in Fig. 5. Although the differences in analyzing power distributions for the two reaction types are not as drastic as those shown in Fig. 4, it is nevertheless clear that the combined mechanism provides much better agreement with the experimental data. 


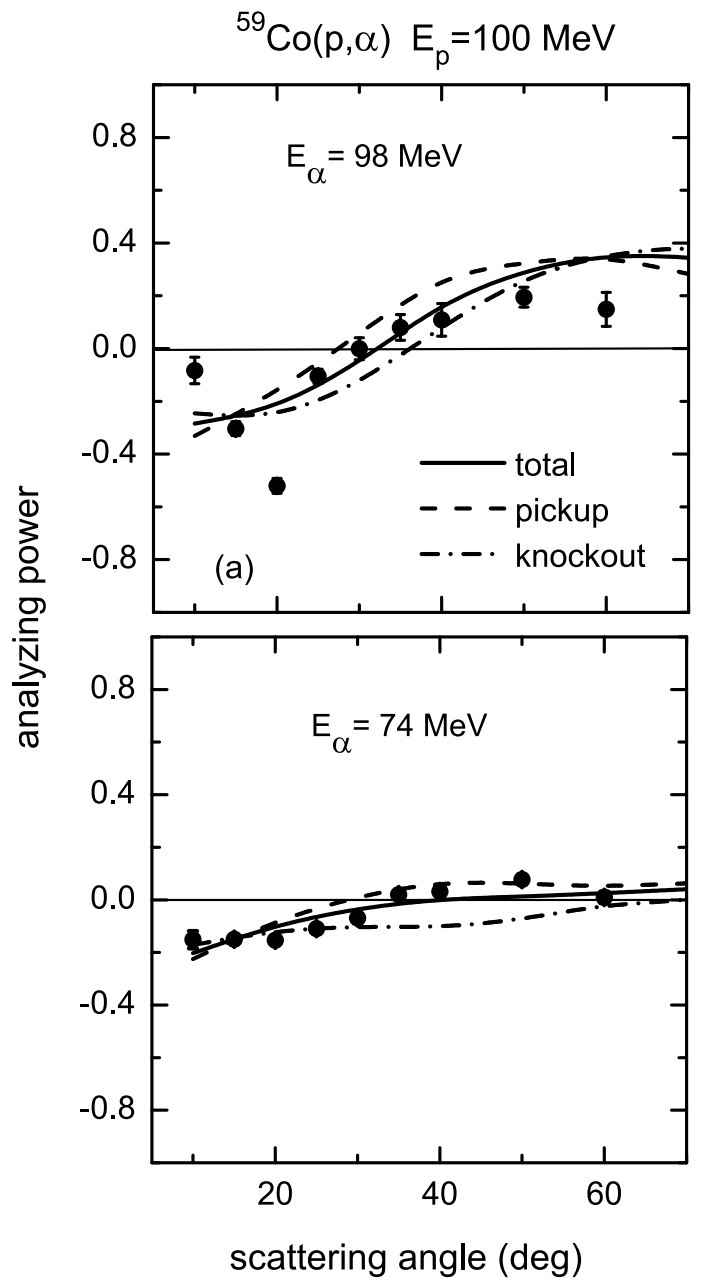

Figure 5. Analyzing power distributions for the reaction ${ }^{59} \mathrm{Co}(p, \alpha)$ at an incident energy of $100 \mathrm{MeV}$. Emission energies are as indicated. The curves correspond to FKK results for a terminating pickup mechanism (dashed curves), knockout (dasheddot curves) or combined values (continuous curves). The displayed results are a subset selected from Ref. [4].

\section{Summary and conclusion}

Clearly the impression that the features of pre-equilibrium reactions should be largely insensitive to details of the structure of the target system was wrongly derived from properties of cross sections only. It is true that cross sections depend only weakly on target properties, but the analyzing power reveals additional information. The $(p, \alpha)$ pre-equilibrium reactions are especially interesting because it offers the possibility of either a knockout process or a pickup mechanism as the final step in the multistep chain. How these two processes compete is determined by structure of the target as well as kinematic dynamics of the projectile. This aspect has come under study only fairly recently, and much remains to be investigated. Preequilibrium proton-induced composite particle emission has been studied on relatively few target species, all very close to symmetric neutron-proton configurations. How an expected change in $\alpha$-clustering on the surface of nu- clei varies with increasing asymmetry influences the $(p, \alpha)$ pre-equilibrium reaction mechanism is of interest.

I thank my colleagues Sevdalina Dimitrova (INRNE, Bulgaria), Elena Zemlyanaya and Konstantin Lukyanov (JINR, Dubna, Russia), and JJ van Zyl (Stellenbosch University) for their advice and support of this overview of our published work. This work is based on the research supported in part by the National Research Foundation of South Africa (Grant Numbers 115020).

\section{References}

[1] A.A. Cowley, J.J. van Zyl, S.S. Dimitrova, E.V. Zemlyanaya, and K.V. Lukyanov, Phys. Rev. C 85, 054622 (2012)

[2] S.S. Dimitrova, A.A. Cowley, J.J. van Zyl, E.V. Zemlyanaya, and K.V. Lukyanov, Phys. Rev. C 89, 034616 (2014)

[3] S.S. Dimitrova, A.A. Cowley, E.V. Zemlyanaya, and K.V. Lukyanov, Phys. Rev. C 90, 054604 (2014)

[4] A.A. Cowley, S.S. Dimitrova, E.V. Zemlyanaya, K.V. Lukyanov, and J.J. van Zyl, Phys. Rev. C 93, 034624 (2016)

[5] A.A. Cowley, S.S. Dimitrova, E.V. Zemlyanaya, K.V. Lukyanov, and J.J. van Zyl, EPJ Web of Conferences 107, 08004 (2016)

[6] S.S. Dimitrova, A.A. Cowley, E.V. Zemlyanaya, and K.V. Lukyanov, EPJ Web of Conferences 107, 08005 (2016)

[7] J.J. Griffin, Phys. Rev. Lett. 17, 478 (1966)

[8] F.E. Bertrand and R.W. Peelle, Phys. Rev. C 8, 1045 (1973).

[9] H. Feshbach, A. Kerman, and S. Koonin. Ann. Phys. (NY) 125, 429 (1980)

[10] T. Tamura, T. Udagawa, and H. Lenske, Phys. Rev. C 26, 379 (1982)

[11] H. Nishioka, H.A. Weidenmüller, and S. Yoshida, Ann. Phys. (NY) 183, 166 (1988)

[12] A.J. Koning and J.M. Akkermans, Phys. Rev. C 47, 724 (1993)

[13] R. Bonetti , A.J Koning, J.M. Akkermans, and P.E. Hodgson, Phys. Rep. 247, 1 (1994)

[14] P.D. Kunz and E. Rost, in Computational Nuclear Physics, edited by K. Langanke et al. (SpringerVerlag, Berlin, 1993) Vol. 2, Chap. 5.

[15] A.A. Cowley, G.J. Arendse, J.W. Koen, W.A. Richter, J.A. Stander, G.F. Steyn, P. Demetriou, P.E. Hodgson, and Y. Watanabe, Phys. Rev. C 54, 778 (1996)

[16] A.A. Cowley, G.J Arendse, G.F. Steyn, J.A. Stander, W.A. Richter, S.S. Dimitrova, P. Demetriou, and P.E. Hodgson, Phys. Rev. C, 55, 1843 (1997)

[17] A. Budzanowski, M. Fidelus, D. Filges, F. Goldenbaum, H. Hodde, L. Jarczyk, B. Kamys, M. Kistryn, St. Kistryn, St. Kliczewski, A. Kowalczyk, E. Kozik, P. Kulessa, H. Machner, A. Magiera, B. PiskorIgnatowicz, K. Pysz, Z. Rudy, R. Siudak, and M. Wojciechowski, Phys. Rev. C 80, 054604 (2009) 
[18] A.A. Cowley, EPJ Web of Conferences 38, 13001 (2012)

[19] W.A. Richter, A.A. Cowley, G.C. Hillhouse, J.A. Stander, J.W. Koen, S.W. Steyn, R. Lindsay, R.E. Julies, J.J. Lawrie, J.V. Pilcher, and P.E. Hodgson, Phys. Rev. C 49, 1001 (1994)

[20] C. Kalbach, Phys. Rev. C 37, 2350 (1988)

[21] A.A. Cowley, A. van Kent, J.J. Lawrie, S.V. Förtsch, D.M. Whittal, J.V. Pilcher, F.D. Smit, W.A. Richter, R. Lindsay, I.J. van Heerden, R. Bonetti, and P.E. Hodgson, Phys. Rev. C 43, 678 (1991)

[22] A.A. Cowley, J. Bezuidenhout, S.S. Dimitrova, P.E. Hodgson, S.V. Förtsch, G.C. Hillhouse, N.M. Jacobs, R. Neveling, F.D. Smit, J.A. Stander, G.F. Steyn, and
J.J. van Zyl, Phys. Rev. C 75, 054617 (2007)

[23] A.A. Cowley, G.F. Steyn, S.S. Dimitrova, P.E. Hodgson, G.J. Arendse, S.V. Förtsch, G.C. Hillhouse, J.J. Lawrie, R. Neveling, W.A. Richter, J.A. Stander, and S.M. Wyngaardt, Phys. Rev. C 62, 064605 (2000)

[24] R.Bonetti, F. Crespi and K.-I. Kubo, Nucl. Phys A 499, 381 (1989)

[25] Z. Lewandowski, E. Loeffler, R. Wagner, H.H. Mueller, W. Reichart and P. Schober, Nucl. Phys. A 389, 247 (1982)

[26] H. Sakai, K. Hosono, N. Matsuoka, S. Nagamachi, K. Okada and K. Maeda, H. Shimizu, Nucl. Phys. A 344, 41 (1980) 